\title{
Estudo exploratório das dimensões dos riscos em empreendimentos da construção civil
}

Exploratory study of the dimensions of risks in civil construction projects

Estudio exploratorio de las dimensiones de los riesgos en proyectos de construcción civil

Recebido: 24/05/2021 | Revisado: 30/05/2021 | Aceito: 31/05/2021 | Publicado: 17/06/2021

\author{
Andrey Pimentel Aleluia Freitas \\ ORCID: https://orcid.org/0000-0003-0136-5696 \\ Universidade Federal Fluminense, Brasil \\ E-mail: andreyfreitas@id.uff.br \\ João Alberto Neves dos Santos \\ ORCID: https://orcid.org/0000-0002-4812-6214 \\ Universidade Federal Fluminense, Brasil \\ E-mail: joaoneves@id.uff.br \\ Eliã Pereira de Oliveira \\ ORCID: https://orcid.org/0000-0002-2884-6513 \\ Universidade Federal Fluminense, Brasil \\ E-mail: eliaoliveira@id.uff.br \\ Danilo Rangel Fernandes \\ ORCID: https://orcid.org/0000-0002-0804-0328 \\ Universidade Federal Fluminense, Brasil \\ E-mail: danilorf@id.uff.br \\ Nylvandir Liberato Fernandes de Oliveira \\ ORCID: https://orcid.org/0000-0002-7063-0556 \\ Universidade Federal Fluminense, Brasil \\ E-mail: liberato.finan@id.uff.br
}

\begin{abstract}
Resumo
Este artigo objetiva aprofundar a análise sobre os elementos de risco presentes em empreendimentos da construção civil, identificando, detalhando, avaliando e classificando os eventos mais relevantes presentes nesse ambiente complexo e dinâmico. A questão central destacada no estudo considera a presença de elementos de risco e seus relativos impactos em projetos de construção, sobretudo nos aspectos relacionados ao atendimento de cronogramas e orçamentos. Para responder ao problema citado, foi desenvolvida uma pesquisa exploratória do tipo Survey junto a uma amostragem não probabilística, caracterizada por 105 engenheiros e profissionais que atuam com temas correlatos à gestão de riscos no setor de construção civil brasileira, complementada por uma análise estatística multivariada, sob a estrutura da análise fatorial exploratória. Os resultados apontam para uma distribuição e classificação dos elementos observados, onde são configurados 205 eventos de risco, devidamente classificados por categorias e fatores. Esse detalhamento possibilita auxiliar futuras abordagens que necessitem, por meio do nível de importância, dimensionar e mensurar, de forma mais detalhada, potenciais aspectos de risco presentes em projetos destinados a empreendimentos construtivos.
\end{abstract}

Palavras-chave: Análise fatorial exploratória; Categorias de risco; Eventos de risco; Fatores de risco; Projetos de construção.

\begin{abstract}
This article aims to deepen the analysis of the risk elements present in construction projects, identifying, detailing, evaluating, and classifying the most relevant events present in this complex and dynamic environment. The central issue highlighted in the study considers the presence of risk elements and their relative impacts on construction projects, especially in aspects related to meeting schedules and budgets. To answer the problem mentioned, an exploratory survey of the Survey type was developed together with a non-probabilistic sample, characterized by 105 engineers and professionals who work with themes related to risk management in the Brazilian construction sector, complemented by a multivariate statistical analysis, under the framework of exploratory factor analysis. The results point to a distribution and classification of the observed elements, where 205 risk events are configured, properly classified by categories and factors. This detail makes it possible to assist future approaches that need, through the level of importance, to dimension and measure, in a more detailed way, potential aspects of risk present in projects destined to constructive undertakings.
\end{abstract}

Keywords: Exploratory factor analysis; Risk categories; Risk events; Risk factors; Construction projects. 


\begin{abstract}
Resumen
Este artículo tiene como objetivo profundizar en el análisis de los elementos de riesgo presentes en los proyectos de construcción, identificando, detallando, evaluando y clasificando los eventos más relevantes presentes en este entorno complejo y dinámico. El tema central destacado en el estudio considera la presencia de elementos de riesgo y sus impactos relativos en los proyectos de construcción, especialmente en aspectos relacionados con el cumplimiento de cronogramas y presupuestos. Para responder al problema antes mencionado, se desarrolló una encuesta exploratoria del tipo Encuesta junto con un muestreo no probabilístico, caracterizado por 105 ingenieros y profesionales que trabajan con temas relacionados con la gestión de riesgos en el sector de la construcción civil brasileña, complementado con un análisis estadístico multivariado., en el marco del análisis factorial exploratorio. Los resultados apuntan a una distribución y clasificación de los elementos observados, donde se configuran 205 eventos de riesgo, debidamente clasificados por categorías y factores. Este detalle permite asistir a futuros enfoques que necesiten, a través del nivel de importancia, dimensionar y medir, de manera más detallada, los potenciales aspectos de riesgo presentes en proyectos orientados a emprendimientos constructivos.
\end{abstract}

Palabras clave: Análisis factorial exploratorio; Categorías de riesgo; Eventos de riesgo; Factores de riesgo; Proyectos de construcción.

\title{
1. Introdução
}

A gestão de riscos apresenta alta relevância no gerenciamento de projetos construtivos, requerendo uma abordagem detalhada dos fatores críticos presentes nos ambientes interno e externo das organizações, de modo mapear e controlar seus potenciais impactos gerados aos processos produtivos.

Em geral, as dificuldades relacionadas ao contexto de riscos estão presentes no cotidiano da construção civil e são percebidas em diversos setores da economia. Governos, entidades de classe, mercado de seguros, instituições de crédito e a sociedade em geral, que constituem um amplo bloco de stakeholders que se defronta com incertezas nessa área, sejam na obtenção das licenças ambientais, referentes à qualidade dos materiais utilizados, inerentes ao gerenciamento dos contratos (Wang \& Yuan, 2011) ou à gestão dos recursos. Enfim, todos esses agentes econômicos sofrem com eventos indesejados que impactam diretamente suas tomadas de decisões, em aspectos associados a qualidade, prazos e custos das obras realizadas.

No campo acadêmico, a literatura destaca a dificuldade para se identificar adequadamente, nos projetos de construção civil, os componentes dos riscos especulativos e suas relativas incertezas frente à expectativa de perdas para os contratantes de seguros. As variáveis mais comuns na mensuração dos riscos em projetos de construção civil envolvem aspectos técnicos.

No referencial teórico, constatou-se que algumas variáveis não são consideradas na mensuração dos riscos. Cita-se, como exemplo, a possibilidade de surgir um novo plano econômico que possa afetar o poder de compra de novos mutuários interessados em adquirir o imóvel em construção. Esse fato tende a provocar uma inadimplência que, em volumes incompatíveis ao projeto, poderia paralisar a obra por falta de fluxo de caixa suficiente para manutenção dos processos construtivos e/ou investimentos.

Dessa forma, muitas empresas do setor de construção contratam apólices de seguro, com base em valores fixados por parâmetros gerados pela experiência e orientação dos profissionais de seguros, frente aos riscos assumidos para o financiamento e gestão das obras. No entanto, esse modelo segue critérios subjetivos de análise, apresentando baixa consistência em sua fundamentação.

Sob esse contexto e considerando o estágio atual, no qual as práticas brasileiras se difundem no setor da construção, tem-se como questão de estudo, abordar a temática relacionada à análise dos elementos de risco envolvidos num empreendimento de construção civil. Do ponto de vista científico, o problema de pesquisa emerge ao se considerar que este trabalho pretende analisar, em uma estrutura exploratória, a relação entre gestão de riscos e gerenciamento de projetos de construção, orientada por duas questões principais: (1) Como identificar e organizar os elementos de risco, de modo a auxiliar na eficácia das ações do gerenciamento de riscos? (2) Qual a dimensão de relevância dos eventos de risco identificados em projetos de empreendimentos na construção civil? 
Baseando-se nesse panorama, este artigo tem por objetivo analisar a configuração dos elementos de risco presentes em empreendimento da construção civil, identificando, avaliando e classificando os eventos mais relevantes. Para tal, serão abordados os eventos de risco associados aos projetos construtivos de empreendimentos, identificando seus respectivos níveis de criticidade, além de agrupá-los conforme seus níveis de estrutura, sobretudo relacionados ao cumprimento de cronogramas e orçamentos.

A abordagem desta pesquisa é justificada por três questões centrais: (i) Examinar um tema ainda não adequadamente identificado na literatura, em pesquisas que abordem quais seriam os principais fatores de avaliação de risco para empresas do setor da construção no Brasil; (ii) Discutir um tema que, em função dos significativos valores de custos, implica em uma gestão severa desses gastos, fazendo necessário estabelecer um modelo de gestão dos riscos à altura de sua importância; e (iii) Proporcionar conhecimentos indispensáveis à tarefa de gestão de riscos, tornando-se extremamente útil ao desenvolvimento de um modelo que permita a avaliação da probabilidade de ocorrência dos fatores de risco que irão formar os riscos de empreendimentos imobiliários.

A contribuição deste estudo se dá por preencher uma lacuna hoje existente no Brasil sobre a questão dos eventos de risco presentes em empreendimentos de construção civil, além de fomentar uma perspectiva interdisciplinar, cujo foco reside na discussão de uma problemática prática que se circunscreve na intersecção multidisciplinar entre as áreas de Engenharia, Arquitetura, Urbanismo, Construção e Administração. Assim, os resultados desta pesquisa contribuem para dar conhecimento sobre um assunto implícito ao alvo da construção civil, já que os empreendimentos imobiliários estão suscetíveis a diversos fatores de risco que dificultam ainda mais a realização dos investimentos, possuindo uma relação muito próxima com a gestão das atividades de engenharia civil, de arquitetura e construção.

\section{Fundamentação Teórica}

A fundamentação bibliográfica deste trabalho foi estruturada e baseada em pesquisas científicas relacionadas a diversas abordagens de riscos em projetos construtivos. Em uma análise mais detalhada, identificou-se que poucas pesquisas trataram diretamente os conceitos de classificação dos níveis de risco, sobretudo em empreendimentos de construção civil, fato que destaca a relevância do tema.

As atividades relacionadas ao setor da construção apresentam características muito inclinadas a diferentes aspectos de risco. As incertezas vão além das atividades delimitadas pelos projetos. Assim, o êxito de um projeto decorre, principalmente do nível de risco associado a ele, em alinhamento com as diferentes partes contratantes, como empreiteiros, subcontratados, fornecedores, proprietários (Freitas et al. 2019).

Fazen, Klüppelberg \& Menzel, (2014,) relacionam o risco a fenômenos complexos, onde sua abordagem requer um conjunto de competências interdisciplinares. Garvey (2015) acrescenta ao risco possibilidades de perda ou ganho, que possam ser potencializados pela magnitude envolvida. Na prática, modelos de gerenciamento de riscos são adaptativos e elaborados para cenários permeados de incertezas e volatilidades (Bissacot \& Oliveira, 2016). Compreender os riscos e gerenciá-los é premissa básica para atender às necessidades corporativas e controlar seus processos produtivos ou estratégicos.

A partir do ambiente de origem, os riscos podem ser identificados como internos ou externos, (IBGC, 2007; Rezakhani, 2012). Outras linhas de pensamento aprimoram este tratamento, conceituando os riscos internos para os que atingem apenas ao empreendimento analisado, onde os gestores possuem responsabilidade direta por sua identificação e pelas ações mitigadoras. Como exemplo desta definição, podem ser citados os riscos contratuais, alguns riscos sociais e ambientais. Nesta linha de pensamento, os riscos externos, são os que podem afetar qualquer atividade econômica e se originam no ambiente externo da empresa, de modo independente da vontade ou do ato de gestão do responsável. Em regra, são associados 
a fenômenos macroeconômicos ou políticos como, por exemplo, a categoria dos riscos políticos, alguns riscos ambientais, sociais etc. (Oliveira, 2018).

Diversos arranjos de gerenciamento de risco foram elaborados por vários pesquisadores. Iqbal et al. (2015) desenvolveram uma estrutura de gestão de risco para relatar a importância de identificar os tipos de riscos e a eficácia das técnicas praticadas por projetos de construção. Outros pesquisadores recomendam a utilização de sistemas de gerenciamento de riscos sob a forma proativa, identificando a probabilidade de ocorrência e agindo de imediato para mitigar as situações indesejadas aos projetos. (Ferreira et al, 2013)

$\mathrm{Na}$ análise dos riscos técnicos, que apresenta maior incidência de ocorrências na construção civil nacional, Marcelli (2007) enfatiza o surgimento de sinistros sobre obras de terra, relacionados a problemas diversos em aterros sobre solo fraco, inclinado, impróprio ou mal compactado ou falhas na execução de estacas. Reformas de reforço em fundações ou sinistros por falhas no projeto estrutural, também são citadas pelo autor.

Nadoushani, Hammad \& Akbarnezhad (2018) destacam as limitações de ordem técnica em equipamentos, como fatores que impactam os custos do setor, em âmbito internacional. Zou et al (2007), destacam os fatores de risco relacionados na elaboração de projetos na China. De acordo com Ferreira et al (2013) é indicativo considerar a probabilidade de eventos de risco ao longo dos projetos, pois diversos níveis de incerteza tendem a impactá-los.

Riscos de cancelamento e de prejuízos em obras são relacionados com casos de corrupção, amplamente discutidos atualmente na sociedade brasileira devido a Operação Lava-Jato. Fato constatado pela elaboração do Guia Ética e Compliance na Construção (CBIC, 2016), que orienta as construtoras locais. Destacam-se ainda, os aspectos de regulamentação conjecturados na Lei n ${ }^{\circ} 12.846$ (Brasil, 2013), intitulada Lei Anticorrupção, em vigência desde janeiro de 2014 (Freitas et al, 2019; Oliveira, 2018).

Ao longo da crise econômica que enfraqueceu parte das nações europeias, no período de 2010 a 2012, em especial no setor da construção civil, Silva (2012) identificou que apenas 27\% das empresas do Reino Unido adotavam uma política de gerenciamento de riscos rigorosa em seus projetos. Diante desse cenário, desenvolveu um guia de procedimentos de riscos, direcionado à seleção de projetos. Nessa mesma linha, outras pesquisas seguiram esse conceito e elaboraram guias e normas de referência para a orientação do gerenciamento dos riscos em processos (Caiado, 2015).

Alguns autores como Silva (2012) e Pinca \& Rozzeto (2018), entendem que as categorias de risco contratuais, políticos, econômicos e financeiros são pouco exploradas. Constatou-se ainda que, outros fatores de risco apresentam elevada dimensão de impacto, como a instabilidade política, os procedimentos contratuais demasiadamente burocráticos e a falta de infraestrutura adequada.

Os principais elementos de risco são elencados e discutidos por diversos autores (Fugar \& Agyakwah, 2010; Gad et al., 2011; Rezakhani, 2012; Ramanathan, Nakayanan, \& Idrus, 2012; Muya-Mundia, Sichombo, \& Shakantu, 2013; Najafabadi \& Pimplikar, 2013; Rosenfeld, 2014; González et al., 2014; Muianga, Granja, \& Ruiz, 2015; Pinca \& Rozzetto, 2018; Freitas, 2018), que os relacionam ao aumento de custos e atrasos em empreendimentos de construção civil, dentre outros impactos.

Muitos autores citam ainda questões relacionadas à influência dos fatores de risco nas categorias de risco econômico, financeiro, político, contratuais, legais e técnicos. Destacam-se também a falta de recursos de financiadores dos projetos, problemas relacionados com a falta de liderança, relacionamentos com o contratante, falhas com a comunicação entre projetista e contratantes do projeto, causas ambientais e outras. (Le-Hoai \& Lee, 2008; Ramanathan, Nakayanan, \& Idrus, 2012; Silva, 2012; Hwang et al., 2013; Fallahnejad, 2013; Rahman, Memon, \& Karim, 2013. Liu et al., 2016). Aspectos dos riscos de origem contratual, de entidades regulamentadoras ou de forte influência na definição de procedimentos e práticas de Compliance praticadas pelas empresas de construção civil estrangeiras e brasileiras, devem ainda ser considerados (Wang \& Yuan, 2011; Ricardino et al., 2013). 
Os elementos de risco discutidos por este artigo tomam como referência a estruturação dos elementos de risco formulada por Oliveira (2018), que foram devidamente segregados, agrupados e organizados, conforme os diferentes níveis dimensão, configurados por categorias, fatores e eventos de risco.

\section{Metodologia}

O estudo contemplado neste artigo adota uma metodologia baseada na pesquisa exploratória, onde as estratégias escolhidas seguem a abordagem de métodos mistos, que resulta de um sistema de coleta e análise de dados, combinado com técnicas quantitativas e qualitativas em um mesmo desenho de pesquisa. Essa tarefa exige que o pesquisador esteja familiarizado em trabalhar com a análise qualitativa e quantitativa de dados textuais e numéricos em um único estudo (Creswell, 2012).

Para atender os objetivos propostos, o método adotado pela pesquisa foi constituído por várias etapas que possibilitaram o desenvolvimento deste artigo, norteando e fundamentando a sequência dos procedimentos metodológicos elencados, conforme mostra a Figura 1.

Figura 1 - Protocolo da pesquisa.

Fonte: Autores (2021).

A metodologia proposta por esta pesquisa destaca três pontos que serão desenvolvidos na ordem a seguir: (i) definição e identificação da amostra; (ii) organização, delimitação da pesquisa e coleta dos dados; e (iii) análise dos dados coletados.

Esta pesquisa utilizou uma amostragem não probabilística por conveniência (Farrokhi \& Mahmoudi-Hamidabad, 2012), dentro das definições descritas por Guimarães (2008), formada por 105 profissionais atuantes no setor da construção civil do Brasil, com vivência em gerenciamento de riscos. O tamanho mínimo da amostra foi dimensionado com base no critério de Leech, Barret \& Morgan (2005), conforme mostrado matematicamente pela Equação 1.

$$
\text { Tamanho da amostra }=(n-p)>50 \rightarrow(105-43)>50 \text { (Equação 1) }
$$

Onde:

$\mathrm{n}=$ Tamanho da amostra.

$\mathrm{p}=$ Quantidade de variáveis presentes.

A partir da definição do tamanho mínimo da amostra, foram considerados, com base na literatura, os níveis restritivos condicionantes para uma abordagem significativa dos fatores de carregamento.

\subsection{Organização dos elementos de risco}

A pesquisa foi organizada a partir de uma fase preliminar, caracterizada pela elaboração de entrevistas por pautas (semiestruturada ou semidirigida). Para Britto \& Feres Júnior (2011), estes tipos de entrevistas representam certo grau de 
estruturação, fundamentada de acordo com pontos de interesses que o entrevistador vai explorando ao longo do seu curso. Em seguida, foi feita a aplicação de um questionário aberto, com 10 questões e estruturado por pautas.

Este questionário inicial foi apresentado a um grupo de 21 respondentes, qualificados como profissionais relacionados ao segmento objeto da pesquisa com atuação superior a 20 anos em sistema de risco. Seus resultados serviram como direcionador para a identificação dos elementos de risco presentes em projetos de empreendimentos de construção civil no Brasil. Nesta fase se inclui ainda a pesquisa bibliográfica sobre os elementos de risco, discutidos no meio acadêmico.

$\mathrm{Na}$ fase final do estudo, foi utilizado um segundo questionário estruturado, aplicado em um grupo de 105 respondentes, caracterizado por profissionais com graduação superior em engenharia e significativa experiência profissional e/ou acadêmica em relação ao tema investigado.

O questionário foi estruturado de modo a contemplar os elementos de risco em três níveis: i) Nível 1 - 9 categorias de risco (1 a 9); ii) Nível 2 - 43 fatores de risco (1.1 a 9.11); e iii) Nível 3 - 205 eventos de risco (1.1.1 a 9.11.5). Suas afirmativas foram fundamentadas sob uma escala Likert, conforme mostra o Quadro 1.

Quadro 1 - Escala de respostas da pesquisa final.

\begin{tabular}{|c|c|c|c|c|}
\hline $\begin{array}{c}\text { Discordo completamente } \\
\text { (DC) }\end{array}$ & $\begin{array}{c}\text { Discordo } \\
\text { (D) }\end{array}$ & $\begin{array}{c}\text { Não concordo, nem discordo } \\
\text { (N) }\end{array}$ & $\begin{array}{c}\text { Concordo } \\
\text { (C) }\end{array}$ & $\begin{array}{c}\text { Concordo completamente } \\
\text { (CC) }\end{array}$ \\
\hline 1 & 2 & 3 & 4 & 5 \\
\hline
\end{tabular}

Fonte: Autores (2021).

A escala Likert é utilizada para estimar o grau de concordância do respondente em relação a cada afirmação (Aaker et al, 2004), onde escores à esquerda descrevem um argumento negativo e à direita, argumentos com maiores níveis de positividade (Hayes, 1997).

A análise proposta por esta pesquisa utilizou a organização dos elementos de risco definida por Oliveira (2018) e foi delimitada somente aos eventos de risco identificados ao longo do estudo. Importante destacar que o "nível de risco 1 Categorias de Risco" e o "nível de risco 2 - Fatores de Risco" foram identificados pela pesquisa bibliográfica, conforme o resumo mostrado no Quadro 2, onde os níveis de risco apresentados surgem de situações recorrentes, identificadas no setor da construção civil em vários países e citadas por vários autores.

Quadro 2 - Categorização dos riscos do setor de construção.

\begin{tabular}{|l|l|}
\hline Categorias de Risco & Fatores de Risco \\
\hline \multirow{4}{*}{ 1. Políticos } & 1.1 Instabilidade política \\
\cline { 2 - 2 } & 1.2 Falta de incentivos do governo \\
\cline { 2 - 2 } & 1.3 Estrutura política fragmentada \\
\cline { 2 - 2 } 2. Legais & 2.4 Realização de eleições \\
\cline { 2 - 2 } & 2.1 Alterações da legislação \\
\cline { 2 - 2 } & 2.3 Complexidade do Sistema Legal \\
\hline \multirow{4}{*}{ 3. Econômicos } & 3.1 Flutuação da inflação e da taxa de juros \\
\cline { 2 - 2 } & 3.2 Instabilidades da economia \\
\cline { 2 - 2 } & 3.3 Flutuação do valor de troca da moeda \\
\hline \multirow{3}{*}{ 4. Financeiros } & 4.1 Impostos \\
\cline { 2 - 2 } & 4.2 Dificuldade no acesso a seguros \\
\hline
\end{tabular}




\begin{tabular}{|c|c|}
\hline & 4.3 Dificuldade no acesso ao crédito \\
\hline & 4.4 Corrupção \\
\hline & 4.5 Reduzida capacidade financeira do dono da obra \\
\hline & 4.6 Reduzida capacidade financeira do empreiteiro \\
\hline & 4.7 Reduzida capacidade financeira dos subempreiteiros \\
\hline \multirow{2}{*}{ 5. Sociais } & 5.1 Conflitos interpessoais (guerras, desordem, revoltas etc.) \\
\hline & 5.2 Conflitos intrapessoais (mentalidade, educação, civismo, comunicação, \\
\hline \multirow{6}{*}{ 6. Mercado } & 6.1 Elevada concorrência \\
\hline & 6.2 Reduzida capacidade de inovação tecnológica \\
\hline & 6.3 Reduzida qualidade dos materiais de construção \\
\hline & 6.4 Reduzida qualidade e elevado custo da mão de obra \\
\hline & 6.5 Reduzida qualidade dos equipamentos de construção \\
\hline & 6.6 Indisponibilidade de subempreiteiros \\
\hline \multirow{5}{*}{ 7. Ambientais (Naturais) } & 7.1 Condições climáticas adversas \\
\hline & 7.2 Condições do solo imprevistas \\
\hline & 7.3 Cheias e enchentes \\
\hline & 7.4 Incêndios \\
\hline & 7.5 Terremotos e abalos sísmicos \\
\hline \multirow{2}{*}{ 8. Contratuais } & 8.1 Tipo de contrato e "preço base" reduzido \\
\hline & 8.2 Prazo reduzido \\
\hline \multirow{11}{*}{ 9. Técnicos } & 9.1 Pouca experiência da equipe projetista \\
\hline & 9.2 Deficiências e /ou falhas de comunicação entre as partes \\
\hline & 9.3 Atrasos na aprovação dos projetos e da regulamentação \\
\hline & 9.4 Erros de projeto \\
\hline & 9.5 Sucessivas alterações aos projetos \\
\hline & 9.6 Informações incompletas \\
\hline & 9.7 Deficiências nas informações para elaboração da proposta \\
\hline & 9.8 Elevada complexidade do projeto \\
\hline & 9.9 Falta de experiência em projetos similares \\
\hline & 9.10 Sinistros em pontos críticos da fase de execução \\
\hline & 9.11 Obras de recuperação ou de reformas sucessivas \\
\hline
\end{tabular}

Fonte: Autores (2021).

\section{Resultados e Discussão}

Os dados obtidos pela fase preliminar indicaram a percepção dos entrevistados frente aos elementos de risco mais significativos e suas respectivas causas. Estas, foram aprofundadas na fase final da pesquisa, resultando no nível 3 da classificação dos riscos (eventos de risco).

Os 21 respondentes da fase preliminar, foram instados a citar três eventos de risco mais relevantes para a construção civil, dentro da abrangência da pesquisa, que foram em seguida associadas às categorias de risco. Os resultados mostram que 58\% dos participantes citaram eventos associados à categoria de riscos técnicos, destacando a complexidade e os diversos riscos de projeto; $15 \%$ das respostas citaram os riscos de mercado, com destaque para a baixa qualidade da mão de obra, do material utilizado e dos equipamentos. Outras categorias de risco também foram apontadas. A Tabela 2 mostra as cinco categorias mais citadas. 
Tabela 1 - Percepção sobre os riscos mais importantes - pesquisa preliminar.

\begin{tabular}{|c|c|c|}
\hline Riscos mais importantes associados às categorias & Totais & Representação \\
\hline Riscos Técnicos (complexidade da construção) & 35 & $58 \%$ \\
\hline Riscos do Mercado (qualidade: da mão de obra, do material, de equipamentos) & 9 & $15 \%$ \\
\hline Riscos Sociais (greves e conflitos) & 7 & $12 \%$ \\
\hline Risco Ambiental & 6 & $10 \%$ \\
\hline Riscos Legais & 3 & $5 \%$ \\
\hline Total de Respostas & 60 & $100 \%$ \\
\hline
\end{tabular}

Fonte: Autores.

Essas informações contribuíram para a estruturação do questionário apresentado aos 105 respondentes da fase final do estudo, onde foi aplicada a Análise Fatorial Exploratória (AFE), objetivando.

Foi realizada uma Análise Fatorial Exploratória (AFE) com o objetivo de avaliar a estrutura fatorial presente entre as variáveis observadas. A análise foi implementada utilizando uma matriz policórica, sob a escala Likert, e pelo método de extração Robust Diagonally Weighted Least Squares (RDWLS) (Hauck-Filho, Teixira, Machado, \& Bandeira, 2012).

A decisão sobre o número de fatores a serem retidos foi caracterizada através da técnica da Análise Paralela, com permutação aleatória dos dados observados e, por fim, aplicada a rotação Robust Promin (Timmerman, \& Lorenzo-Seva, 2011; Lorenzo-Seva \& Ferrando, 2019).

Aaker et al (2004) destacam que a análise fatorial possibilita a combinação de variáveis que resultam em novos fatores. Convergindo a esse entendimento, Hair et al (2014) definem que um fator explica a combinação linear entre variáveis originais. A literatura destaca a utilização da análise fatorial para a verificação das correlações entre variáveis por meio da identificação dos fatores comuns. Com essa finalidade, uma Análise Fatorial Exploratória foi aplicada aos dados coletados, resultando na classificação das variáveis em níveis categóricos, conforme os agrupamentos identificados e apresentados na Tabela 2.

Tabela 2 - Síntese dos resultados das categorias de risco - nível 1.

\begin{tabular}{lccc}
\hline Categorias de Risco $\left(1^{\circ}\right.$ Nível $)$ & Ambiente & Alfa de Cronbach & KMO \\
\hline $1-$ Riscos Políticos & Externo & 0,841 & 0,729 \\
$2-$ Riscos Legais & Externo & 0,885 & 0,854 \\
$3-$ Riscos Econômicos & Externo & 0,865 & 0,794 \\
$4-$ Riscos Financeiros & Misto & 0,936 & 0,841 \\
$5-$ Riscos Sociais & Misto & 0,942 & 0,864 \\
$6-$ Riscos de Mercado & Misto & 0,926 & 0,831 \\
$7-$ Riscos Ambientais & Interno & 0,935 & 0,796 \\
$8-$ Riscos Contratuais & Interno & 0,874 & 0,781 \\
$9-$ Riscos de Ordem Técnica & Interno & 0,981 & 0,779 \\
\hline
\end{tabular}

Fonte: Autores.

Os valores presentes na Tabela 2 conferem a validade do conteúdo e consistência de confiabilidade ao instrumento, através dos testes de KMO (Kaiser-Meyer-Olklin) e do coeficiente Alfa de Crombach. A consistência verificada foi 
significante, pois apresentou escore superior a 0,60 .

Para a aplicação dos testes, os elementos estudados foram agrupados por níveis, definidos como: categorias de risco (nível 1), fatores de risco (nível 2) e eventos de risco (nível 3). Com base nessa organização, o primeiro nível de riscos contempla nove categorias:

1. Riscos Políticos - Identificam os fatores formados a partir de eventos de risco relacionados à interferência de decisões governamentais ou eleitorais (Silva, 2012);

2. Riscos Legais - Fatores que se originam a partir de eventos de risco relacionados à aspectos da legislação ou regulamentação em vigor (Pinca \& Rozzetto, 2018);

3. Riscos Econômicos - Contemplam os fatores com eventos de risco associados a aspectos da política econômica empregada (Pinca \& Rozzetto, 2018);

4. Riscos Financeiros - Referem-se aos fatores com eventos de risco conectados aos diversos aspectos da gestão financeira do negócio ou dos projetos e incluem a parte da tributação, seguros, crédito, fluxo de caixa e corrupção (Nutakor, 2007; CBIC, 2016; Pinca \& Rozzetto, 2018);

5. Riscos Sociais - Dizem respeito aos fatores com eventos de risco relacionados às estruturas sociais em que a empresa ou o projeto estão inseridos (Bu-Qammaz, 2007; González et al., 2014; Pinca \& Rozzetto, 2018);

6. Riscos de Mercado - Estão associados aos fatores que se originam a partir de eventos de risco relacionados com aspectos do segmento de mercado ao qual a empresa ou o projeto estão inseridos. Considera os possíveis efeitos da participação dos concorrentes, da inovação tecnológica, da qualidade da mão de obra ou dos materiais empregados (BuQammaz, 2007);

7. Riscos Ambientais - Reportam os fatores com eventos de risco associados a aspectos climáticos, de solo, de chuvas ou cheias, de terremotos e ainda os riscos de incêndio. Esta categoria é citada por Shehu, Endut, \& Akintoye (2014) e por Shi et al (2017) como os principais fatores causadores de atrasos e incremento de custos;

8. Riscos Contratuais - Fatores com eventos de Risco associados a aspectos da contratação dos serviços de construção civil como os riscos relativos à formação do preço - base ao prazo que afetam o cronograma da obra. Tal pensamento é também partilhado por Ricardino et al. (2013) em estudos sobre problemas causados por questões contratuais.

9. Riscos de Ordem Técnica - Esta categoria, conforme destaca Zou et al (2007), contempla os fatores de risco técnicos relacionados a projetos. Nesta pesquisa, o foco nos fatores e eventos de Risco são associados aos principais aspectos da construção e que incluem a parte do projeto, da execução e do controle da obra. (Bu-Qammaz, 2007; Marcelli, 2007; Nutakor, 2007; González et al., 2014; Le-Hoai \& Lee, 2008; Pinca \& Rozzetto, 2018).

Os resultados encontrados, no questionário da fase final da pesquisa indicaram, na primeira coluna, a relação nominal das Categorias (nível 1), dos Fatores de Risco (nível 1.1) e dos Eventos de Risco (nível 1.1.1). A classificação adotada seguiu uma lógica de agrupamento para os riscos estudados da seguinte forma:

$1^{\circ}$ Categorias de Risco nível 1 - constitui os elementos de um mesmo tipo de risco, distribuídos em 9 grandes grupos: (1) Riscos Políticos, (2) Riscos Legais, (3) Riscos Econômicos, (4) Riscos Financeiros, (5) Riscos Sociais, (6) Riscos de Mercado, (7) Riscos Decorrentes de Problemas Ambientais, ou Causas Naturais, ou apenas, Riscos Ambientais, (8) Riscos Contratuais e (9) Riscos de Ordem Técnica;

$2^{\circ}$ Fatores de Risco (nível 2) - compreendem um primeiro subgrupo de elementos que agregam os desdobramentos do grupo principal de riscos. Como, por exemplo, a Instabilidade Política que resulta de uma série de eventos de risco, relacionados a forma de condução dos governos ou aos cenários de instabilidade política;

$3^{\circ}$ Eventos de Risco (nível 3) - menores itens que deflagram situações primárias de riscos ao projeto de empreendimento de construção civil e compõem a estrutura do nível acima, configurando aspectos por menores, como: 
prejuízos, atrasos etc.

A delimitação desta pesquisa está na abordagem aos eventos de risco, que foram identificados e classificados a partir dos fatores de risco presentes na literatura e do questionário da fase preliminar, com base na amplitude das categorias (ou grupos) de risco presentes na literatura. Para resumir a análise, são relacionados os três níveis da dimensão dos riscos, destacando os eventos de risco de maior e menor relevância, encontrados na pesquisa.

Os resultados encontrados sobre as respostas de cada categoria foram satisfatórios, confirmando e validando o constructo. A partir dos escores obtidos, foi possível identificar os eventos de risco de maior e menor relevância em cada categoria de risco, conforme destacado pela Tabela 3.

Tabela 3 - Classificação dos níveis de risco, conforme extremidade dos valores dos eventos de risco.

\begin{tabular}{|c|c|c|c|c|}
\hline \multirow{2}{*}{$\begin{array}{c}\text { Categoria } \\
\left(1^{\circ} \text { nível }\right)\end{array}$} & \multirow{2}{*}{$\begin{array}{c}\text { Fator } \\
\left(2^{\circ} \text { nível }\right)\end{array}$} & \multirow{2}{*}{$\begin{array}{c}\text { Evento } \\
\left(3^{\circ} \text { nível }\right)\end{array}$} & \multicolumn{2}{|c|}{ Relevância } \\
\hline & & & Maior & Menor \\
\hline \multirow[b]{2}{*}{ 1. Político } & 1.2 Falta de incentivos do governo & $\begin{array}{l}\text { 1.2.1 Reduções de financiamentos públicos, na elaboração } \\
\text { de seus projetos. }\end{array}$ & 4,170 & \\
\hline & 1.3 Estrutura política fragmentada & $\begin{array}{l}\text { 1.3.3 Seus ideais e políticas de gestão virem a ser diferentes } \\
\text { das praticadas pelo governo a ponto de afetarem a } \\
\text { rentabilidade de seus projetos. }\end{array}$ & & 3,410 \\
\hline \multirow{2}{*}{ 2. Legais } & 2.3 Complexidade do Sistema Legal & 2.3.3 Contingências ambientais legais. & 4,190 & \\
\hline & 2.1 Alterações da legislação & 2.1.3 Cancelamentos de projetos. & & 3,629 \\
\hline \multirow[b]{2}{*}{ 3. Econômicos } & 3.2 Instabilidades da economia & 3.2.4 Redução de obras públicas. & 4,305 & \\
\hline & $\begin{array}{l}\text { 3.3 Flutuação do valor de troca da } \\
\text { moeda }\end{array}$ & 3.3.2 Cancelamento de novos projetos. & & 3,692 \\
\hline \multirow{2}{*}{ 4. Financeiros } & $\begin{array}{l}\text { 4.6 Reduzida capacidade financeira } \\
\text { do empreiteiro }\end{array}$ & 4.6.1 Ocorrerem atrasos no cronograma. & 4,324 & \\
\hline & 4.2 Dificuldade no acesso a seguros & $\begin{array}{l}\text { 4.2.2 Vir a ocorrer o cancelamento de novas apólices de } \\
\text { seguros, caso ocorram acidentes naturais em seus projetos. }\end{array}$ & & 3,288 \\
\hline
\end{tabular}

5.1 Conflitos interpessoais (guerras, desordem, revoltas etc.)

5.1.5 Perda de trabalhadores, equipamentos ou materiais. $\quad 4,010$

5.2 Conflitos intrapessoais (mentalidade, educação, civismo, 5.2.3 Atrasos ao projeto por conflitos interpessoais entre trabalhadores. comunicação, cultura etc.)

6.4 Reduzida qualidade e elevado custo da mão de obra

6. Mercado

6.2 Reduzida capacidade de inovação tecnológica 
(Naturais)

\begin{tabular}{|c|c|c|c|c|}
\hline \multirow{2}{*}{ 8. Contratuais } & \multirow{2}{*}{$\begin{array}{l}\text { 8.1 Tipo de contrato e "preço base" } \\
\text { reduzido }\end{array}$} & 8.1.2 Ter seus lucros reduzidos. & 4,125 & \\
\hline & & 8.1.3 Ser forçada a diminuir o padrão de qualidade da obra. & & 3,327 \\
\hline \multirow[b]{2}{*}{ 9. Técnicos } & 9.5 Sucessivas alterações aos projetos & 9.5.2 Aumentos nos custos do projeto. & 4,333 & \\
\hline & $\begin{array}{l}\text { 9.7 Deficiências nas informações para } \\
\text { elaboração da proposta }\end{array}$ & $\begin{array}{l}\text { 9.7.5 Imagem afetada por produzir a obra abaixo do seu } \\
\text { padrão de qualidade }\end{array}$ & & 3,505 \\
\hline
\end{tabular}

Fonte: Autores.

\section{Conclusão}

O método utilizado para a revisão da literatura possibilitou o conhecimento, através da identificação dos eventos que impactam os fatores e categorias de risco, dos principais eventos que apresentam elementos de risco aos projetos de construção civil, bem como seus impactos, relacionados, sobretudo, ao cumprimento de cronogramas e orçamentos.

Os resultados gerais apontam para 205 eventos de risco observados e analisados pela pesquisa, por meio da utilização de técnicas de estatística multivariada. Tais eventos de risco foram caracterizados por ocorrências que, apesar de contempladas por projetos construtivos, podem surgir e ocasionar problemas de atrasos, cancelamentos, aumentos de custos ou prejuízos, além de diversos impactos em questões operacionais, táticas ou estratégicas.

$\mathrm{Na}$ classificação fundamentada por esta pesquisa, os eventos de risco representaram os elementos que influenciam a origem dos fatores de risco que, por sua vez, foram agrupados por gêneros e constituíram a abrangência das categorias de risco.

A construção do conhecimento sobre os questionários fez-se com o apoio teórico sobre o assunto e a identificação dos principais eventos que compõem os elementos de risco em empreendimentos de construção, analisados à luz da Análise Fatorial Exploratória. Daí, com base na percepção dos respondentes, resultaram importantes constatações sobre os eventos de riscos na construção civil, cabendo citar:

(i). Dentro da categoria de Riscos Políticos (1), os fatores relacionados aos riscos de ocorrerem falta de incentivos do governo (1.2) se destacam como os riscos mais relevantes, sendo os eventos de risco decorrentes de reduções de financiamentos públicos, na elaboração de seus projetos (1.2.1) o item com maior relevância dessa categoria. Isso ocorre enquanto o fator de risco de uma estrutura política fragmentada (1.3) causadas por eventos de risco que podem vir a afetar os ideais e políticas de gestão da empresa virem a se tornar diferentes das praticadas pelo governo, a ponto de afetarem a rentabilidade de seus projetos (1.3.3), é considerado muito baixo. Os eventos de risco versados na categoria dos Riscos Políticos e Jurídicos são considerados muito importantes por Oliveira, et al (2021), que citam problemas associados à estrutura política dos governos, à burocracia e corrupção, como fatores influenciadores na implementação dos projetos.

(ii). Sob a perspectiva dos respondentes, verificou-se, ainda, que o risco mais significativo, em todas as nove categorias, reside no Risco Técnico (Categoria 9), de que sucessivas alterações no projeto (Fator 9.5) poderão provocar aumentos nos custos (Evento 9.5.2).

(iii). O menor risco identificado entre todas as categorias foi apontado em Riscos Ambientais ou de Causas Naturais (Categoria 7), de surgirem terremotos ou abalos sísmicos (Fator 7.5) e como consequência, provocar um comprometimento da imagem da empresa (Evento 7.5.5). Fato bastante compreensível, uma vez que este tipo de problema é raro no meio ambiente do território brasileiro. 
Concluiu-se que os eventos de risco quanto ao seu ambiente (internos ou externos), não seguem um padrão único relacionado à categoria em que se encontram. Uma mesma categoria, como observado em Riscos Legais (2), Riscos Financeiros (4), Riscos Sociais (5) e Riscos de Mercado (6), registram eventos de risco classificados no ambiente interno e no ambiente externo.

A pesquisa contribuiu para identificar e organizar em um padrão lógico, os eventos de risco que fazem parte da rotina dos projetos de construção civil. As atividades de gestores de risco podem ser beneficiadas com essas informações, que podem auxiliar no aprimoramento dos controles internos, especificamente na ação de mitigar os eventos de riscos identificados. Outra contribuição relevante pode ser direcionada na busca por racionalizar os critérios e parâmetros adotados por profissionais de seguro, na formulação e mensuração dos riscos previstos para cobertura em apólices.

Para estudos e trabalhos futuros, sugere-se o desenvolvimento de pesquisas de campo direcionadas ao sistema de gerenciamento de riscos em diferentes contextos ou setores, verificando possíveis similaridades entre os níveis e dimensões dos elementos, quando comparados ao ambiente da construção civil.

\section{Referências}

Aaker, D., Kumar, V. \& Day, G. S. (2004) Pesquisa de Marketing. São Paulo: Atlas.

Bissacot, T. C. C. \& Oliveira, S. M. A. C. (2016) Instrumento para o gerenciamento de riscos ambientais. Engenharia Sanitária e Ambiental, v.21, n.2, p 227232. DOI: $10.1590 / \mathrm{S} 1413-41522016140442$

Brasil. (2013) Lei 12.846/2013, de 1 de agosto de 2013. Lei Anticorrupção ou Lei da Empresa Limpa. Brasília: Diário Oficial da União.

Britto Jr, A. \& Feres Jr, N. A. (2011) Utilização da técnica da entrevista em trabalhos científicos. Evidência, v. 7, n. 7, p 237-250.

Bu-Qammaz, A. S., Dikmen, I. \& Birgonul, M. T. (2009) Risk assessment of international construction projects using the analytic network process. Canadian Journal of Civil Engineering, v 36, n. 7, p 1170-1181. DOI: https://doi.org/10.1139/L09-061.

Caiado, R. G. G. (2015) Diretrizes para Maturidade da Gestão de Risco em Projetos de Construção. Dissertação (mestrado) - Universidade Federal Fluminense: Escola de Engenharia, Niterói.

CBIC - Câmara Brasileira da Indústria da Construção. (2016) Guia de Ética e Compliance para Instituições e Empresas do Setor da Construção - Ética e Compliance na Construção. Brasília: CBIC.

Creswell, J. W. (2012) Qualitative Inquiry and Research Design: Choosing Among Five Approaches. Thousand Oaks, CA: Sage.

Fallahnejad, M. H. (2012) Delay Causes in Iran Gas Pipeline Projects. International Journal of Project Management, v. 31, n. 1, p 136-146. DOI: 10.1016/j.ijproman.2012.06.003.

Farrokhi, F. \& Mahmoudi-Hamidabad, A. R. (2012) Rethinking Convenience Sampling: Defining Quality Criteria. Theory and Practice in Language Studies, v. 2, n. 4, p 784-792. DOI:10.4304/tpls.2.4.784- 792 .

Ferreira, B. A. A., Almeida, J. O. R. Leão, P. R. C. \& Silva, N. P. G. (2012) Gestão de Riscos em Projetos: Uma Análise Comparativa da Norma ISO 31000 e o Guia PMBOK. Revista de Gestão e Projetos, v. 4, n. 3, p 46-72. DOI: 10.5585/gep.v4i3.173.

Freitas, A. P. F. (2018) Metodologia de Gerenciamento de Riscos na Indústria da Construção Civil: Aplicação em empresas de micro e pequeno portes. Tese (doutorado) - Universidade Federal Fluminense: Escola de Engenharia, Niterói.

Fugar, F. D. K. \& Agyakwah-Baah, A. B. (2010) Delays in Building Construction Projects in Ghana. Australasian Journal of Construction Economics and Building, v. 10, n. 1/2, p 103-116, 2010.

Garvey, P.R. (2009) Analytical methods for risk management: A systems engineering perspective. Boca Raton: CRC Press, p 1-12.

González, P., Gonzalez, V., Molenaar, K. \& Orozco, F. (2014) Analysis of Causes of Delay and Time Performance in Construction Projects. Journal of Construction Engineering and Management, v. 140, n. 1, 04013027. DOI: 10.1061/(ASCE)CO.1943-7862.0000721.

Guimarães, P. R. B. (2008) Métodos Quantitativos Estatísticos. Curitiba: IESDE Brasil S.A.

Hayes, B. (1997) Measuring Customer Satisfaction: Survey Design, Use, and Statistical Analysis Methods. ${ }^{\mathrm{a}}$ Ed. Milwaukee: Quality Press.

Hair JR, J. F., Black, W. C., Babin, B. J. \& Anderson R. E. (2014) Multivariate Data Analysis. Pearson International Education Limited: Edinburg.

Hauck-Filho, N., Teixeira, M. A. P., Machado, W. L. \& Bandeira, D. R. (2012). Marcadores reduzidos para a avaliação da personalidade em adolescentes. Psico-USF, 17(2), 253-261. 
Hwang, B.G. \& Zhao, X. (2013) Identifying the Critical Factors Affecting Schedule Performance of Public Housing Projects. Habitat International, v. 38, p 214-221. DOI: https://doi.org/10.1016/j.habitatint.2012.06.008

Leech, N., Barret, K. \& Morgan, G. A. (2005) SPSS for intermediate statistics: use and interpretation. Lawrence Erlbaum Associates: New Jersey.

Le-Hoai, L., Lee Y. D. \& Lee J. Y. (2008) Delay and Cost Overruns in Vietnam Large Construction Projects: a comparison with other selected countries. Ksce Journal of Civil Engineering, v. 12, n. 6, p. 367-377. DOI 10.1007/s12205-008-0367-7.

Liu, Y., Liu, Y., Li, H., Fu, X., Guo, H., Meng, R., Lu, W., Zhao, M. \& Wang, H. (2016) Health risk impacts analysis of fugitive aromatic compounds emissions from the working face of a municipal solid waste landfill in China. Environment International, v. 97, p 15-27. DOI: https://doi.org/10.1016/j.envint.2016.10.010.

Lorenzo-Seva, U. \& Ferrando, P. J. (2019). Robust Promin: a method for diagonally weighted factor rotation. Technical report, URV. Tarragona: Spain.

Marcelli, M. (2007) Sinistros na Construção Civil: Causas e Soluções Para Danos e Prejuízos em Obras. São Paulo: Pini.

Muianga, E. A. D., Granja, A. D. \& Ruiz, J. A. (2015) Desvios de custos e prazos em empreendimentos da construção civil: categorização e fatores de influência. Ambiente Construído, v. 15, n. 1. DOI: http://dx.doi.org/10.1590/S1678-86212015000100008.

Muya-Mundia, C. K., Sichombo, B. \& Shakantu, W. (2013) Cost Escalation, Schedule Overruns and Quality Shortfalls on Construction Projects: The Case of Zambia. International Journal of Construction Management, v. 13, n. 1, p 53-68. DOI: https://doi.org/10.1080/15623599.2013.10773205.

Nadoushani, Z. S. M., Hammad, A. W. A. \& Akbarnezhad, A. (2018) Closure to Location Optimization of Tower Crane and Allocation of Material Supply Points in a Construction Site Considering Operating and Rental Costs. Journal of Construction Engineering and Management. v. 144, Issue 11. DOI: https://doi.org/10.1061/(ASCE)CO.1943-7862.0001558

Najafabadi, E. \& Pimplikar, D. (2013) The Significant Causes and Effects of Delays in Ghadir 2206 Residential Project. Journal of Mechanical and Civil Engineering, v. 7, n. 4, p 75-81.

Nutakor G. (2007) Assessing final cost of construction at bid time. Cost Engineering, v. 49, n. 11, p 10-16.

Oliveira, E. P. de, Santos, J. A. N. dos, Freitas, A. P. A. \& Neves, S. F. M. (2021) Corruption in Brazil: Analysis of transparency from the perspective of the civil construction sector. Research, Society and Development, v. 10, n. 5, p e46310515257. DOI: 10.33448/rsd-v10i5.15257.

Oliveira, N. L. F. (2018) Análise de Riscos em Empreendimentos de Construção Civil no Brasil: Um Método de Aferição. Tese (doutorado) - Universidade Federal Fluminense: Escola de Engenharia, Niterói.

Pinca, D. F. \& Rozzetto D. S. (2018) Interrupções em projetos de engenharia consultiva em cenário de desestruturação organizacional. Revista iPecege, v. 4, n. 2, p 54-61. DOI: 10.22167/r.ipecege.2018.2.54.

Rahman, I. A., Memon, A. H. \& Karim, A. T. A. (2013) Relationship between Factors of Construction Resources Affecting Project Cost. Modern Applied Science, v. 7, n. 1, p 67-75. DOI:10.5539/mas.v7n1p67.

Ramanathan, C., Narayanan, S. P. \& Idrus, A. B. (2012) Construction delays causing risks on time and cost - a critical review. Australasian Journal of Construction Economics and Building, v. 12, n. 1, p 37-57, 2012. DOI: https://doi.org/10.5130/AJCEB.v12i1.2330.

Rezakhani, P. (2012) Classifying Key Risk Factors in Construction Projects. The Bulletin of the Polytechnic Institute of Jassy, Construction. Architecture Section, v. 58, n. 2, p 27-38

Ricardino, R., Silva, S., \& Alencar, C. (2013) Causas Frequentes de Reivindicações Contratuais. Revista Engenharia, v. 70, n. 615, p 90-94.

Rosenfeld, Y. (2014) Root-Cause Analysis of Construction-Cost Overruns. Journal of Construction Engineering and Management, v. 140, n. 1, p 04013039. DOI: https://doi.org/10.1061/(asce)co.1943-7862.0000789.

Shehu, Z., Endut, I. R. \& Akintoye, A. (2014) Factors Contributing to Project Time and Hence Cost Overrun in the Malaysian Construction Industry. Journal of Financial Management of Property and Construction, v. 19, n. 1, p 55-75. DOI: https://doi.org/10.1108/JFMPC-04-2013-0009.

Shi, B., Jiang, J., Liu, R., Khan, A. U. \& Wang, P. (2017) Engineering risk assessment for emergency disposal projects of sudden water pollution incidents. Environmental Science and Pollution Research, n 24, p 14819-14833. DOI: 10.1007/s11356-017-9078-2.

Silva V. F. (2012) Análise de Risco na Construção - Guia de Procedimentos para Gestão. Dissertação (mestrado) - Universidade do Porto, Faculdade de Engenharia.

SUSEP - Superintendência de Seguros Privados. (2011) Circular SUSEP nº 519, de 17 de janeiro de 2011. Brasília: SUSEP.

SUSEP - Superintendência de Seguros Privados. (2016) Circular SUSEP n $n^{\circ}$ 540, de 14 de outubro de 2016. Brasília: SUSEP.

Timmerman, M. E. \& Lorenzo-Seva, U. (2011). Dimensionality Assessment of Ordered Polytomous Items with Parallel Analysis. Psychological Methods, 16, 209-220. doi:10.1037/a0023353.

Wang, J. \& Yuan, H. (2011) Factors affecting contractors' risk attitudes in construction projects: Case study from China. International Journal of Project Management, v. 29, n. 2, p 209-219. DOI: 10.1016/j.ijproman.2010.02.006.

Zou, P. X. W., Zhang, G. \& Wang, J. (2007) Understanding the Key Risks in Construction Projects in China. International Journal of Project Management, v. 25, n. 6, p 601-614. DOI: https://doi.org/10.1016/j.ijproman.2007.03.001. 